\title{
Electroencephalographic Features of Temporal Lobe Epilepsy
}

\author{
Mohammed M. Jan, Mark Sadler, Susan R. Rahey
}

\begin{abstract}
Electroencephalography (EEG) is an important tool for diagnosing, lateralizing and localizing temporal lobe seizures. In this paper, we review the EEG characteristics of temporal lobe epilepsy (TLE). Several "non-standard" electrodes may be needed to further evaluate the EEG localization. Ictal EEG recording is a major component of preoperative protocols for surgical consideration. Various ictal rhythms have been described including background attenuation, start-stopstart phenomenon, irregular 2-5 Hz lateralized activity, and 5-10 Hz sinusoidal waves or repetitive epileptiform discharges. The postictal EEG can also provide valuable lateralizing information. Postictal delta can be lateralized in $60 \%$ of patients with TLE and is concordant with the side of seizure onset in most patients. When patients are being considered for resective surgery, invasive EEG recordings may be needed. Accurate localization of the seizure onset in these patients is required for successful surgical management.
\end{abstract}

RÉSUMÉ: Caractéristiques électroencéphalographiques de l'épilepsie temporale. L'électroencéphalographie (EEG) est un outil important pour le diagnostic, la latéralisation et la localisation de l'épilepsie temporale (ET). Dans cet article, nous revoyons les caractéristiques électroencéphalographiques de l'épilepsie temporale. Plusieurs électrodes «non-standard » peuvent être nécessaires pour une évaluation plus poussée de la localisation EEG. L'enregistrement EEG ictal est une composante importante des protocoles préopératoires pour des motifs chirurgicaux. Différents rythmes ictaux ont été décrits, dont l'atténuation du rythme de fond, le phénomène départarrêt-départ, une activité latéralisée irrégulière à $2-5 \mathrm{~Hz}$ et des ondes sinusoïdales à $5-10 \mathrm{~Hz}$ ou des décharges épileptiformes répétitives. L'EEG postictal peut également fournir de l'information précieuse pour la latéralisation. L'activité delta postictale peut être latéralisée chez $60 \%$ des patients atteints d'ET et concorde avec le côté du début de la crise chez la plupart des patients. Quand les patients sont évalués en vue d'une résection chirurgicale, des enregistrements EEG effractifs peuvent s'avérer nécessaires. Le succès de la chirurgie repose sur la localisation exacte du début des crises chez ces patients.

Can. J. Neurol. Sci. 2010; 37: 439-448

Electroencephalography (EEG) remains the single most important physiologic test of the cerebral cortex ${ }^{1,2}$. In temporal lobe epilepsy (TLE), focal interictal and ictal epileptiform discharges help in identifying the involved cortical areas ${ }^{3}$. Several nonepileptiform EEG abnormalities can also be seen in TLE. Scalp and sometimes invasive EEG recordings are needed for accurate localization of the seizure onset. The development of video-EEG monitoring has allowed careful correlation of clinical semiology with simultaneous EEG recordings ${ }^{4}$. This paper will review the EEG characteristics of TLE.

\section{EEG RECORDING}

\section{Scalp EEG recording}

The 10-20 electrode system is an internationally accepted standard method of measurement and application of EEG scalp electrodes ${ }^{5}$. The standard electrodes can detect only up to $58 \%$ of all spikes ${ }^{6}$. Therefore, additional measured and more closely spaced scalp electrodes placed midway between the standard electrodes of the 10-20 system can provide further localization of epileptiform discharges in TLE7. Several "non-standard" electrodes can be used to further evaluate the EEG abnormalities including sphenoidal, nasopharyngeal, anterior temporal

From the Department of Pediatrics (MMJ), Faculty of Medicine, King Abdulaziz University, Jeddah, Saudi Arabia; Division of Neurology and Dalhousie University Medical School (MS); Neuroelectrodiagnostic Unit (SRR), Capital District Health Authority, Halifax, Nova Scotia, Canada.

Received February 1, 2010. Final Revisions Submitted April 12, 2010. Correspondence to: Mohammed M.S. Jan, Department of Pediatrics, Faculty of Medicine, King AbdulAziz University, Box 80215, Jeddah 21589, Kingdom of Saudi Arabia. 
(T1/T2), and surface electrodes applied over the mandibular notch $(\mathrm{MN})$ or zygoma $^{7-9}$.

Sphenoidal electrodes are inserted bilaterally through the skin below the zygomatic arch, 2-3 cm anterior to the tragus and directed posterosuperiorly in the direction of the foramen ovale $^{10}$. This procedure should be performed with local anesthesia by a qualified physician. Flexible wire electrodes, insulated and bared at the tip, are placed about $3 \mathrm{~cm}$ deep by attaching them to the external shaft of a 22 gage special (lumbar puncture like) needle. The needle is then removed leaving the recording electrode in place; the external wire is coiled to relieve tension and taped to the skin.

Nasopharyngeal electrodes are uncomfortable and have not consistently demonstrated superior sensitivity for spike detection when compared to the other non-standard electrodes ${ }^{11,12}$. Therefore, nasopharyngeal electrodes are no longer in widespread use. Sphenoidal, T1/T2, and MN electrodes can record spikes not detected by standard electrodes (Figure 1). Sphenoidal spikes are almost always detected by T1/T2 or MN electrodes, although with slightly lower amplitudes ${ }^{13}$. Recording with multiple non-standard electrodes simultaneously to obtain a more comprehensive evaluation of the electrical field has been recommended ${ }^{7}$.

Few studies have evaluated the ictal (as opposed to interictal) merits of these electrodes or have addressed the issue of montage design for interictal and ictal TLE abnormalities ${ }^{14}$. The ability to perform off-line montage reformatting with digital EEG technology diminishes the importance of initial montage selection. Ives et al studied ictal EEGs in patients with sphenoidal electrodes by using a coronal sphenoidal montage as compared to an anterior-posterior temporal montage ${ }^{14}$. The sphenoidal montage was superior in the detection of ictal EEG changes.

\section{Invasive EEG recording}

Invasive intracranial techniques using subdural or intracerebral depth electrodes can be used to improve diagnostic accuracy if surface EEG recording yields inconclusive or ambiguous results ${ }^{15}$. Recent shifts in surgical candidacy toward younger age groups resulted in increased utility of invasive monitoring especially in patients with atypical clinical semiology or normal imaging studies ${ }^{16}$. Subdural electrodes are inserted surgically to record over the cerebral cortex. Electrode grids are square or rectangular in shape with small platinum or stainless steel disks embedded into soft silastic with several contact points. Different sizes and shapes can be selected dependant on the anatomic area to be covered and require a craniotomy for insertion. Electrode strips, of various sizes, consist of a row of contacts and are usually inserted through a burr hole.

Intracerebral or depth electrodes are used to record from within the temporal lobe using rigid or flexible probes. Multiplelead intracerebral electrodes can be implanted stereotactically (stereoelectroencephalography) to allow sampling from multiple medial (amygdala, hippocampus, entorhinal cortex, insular cortex) and lateral (temporal pole, gyri, parietotemporal and

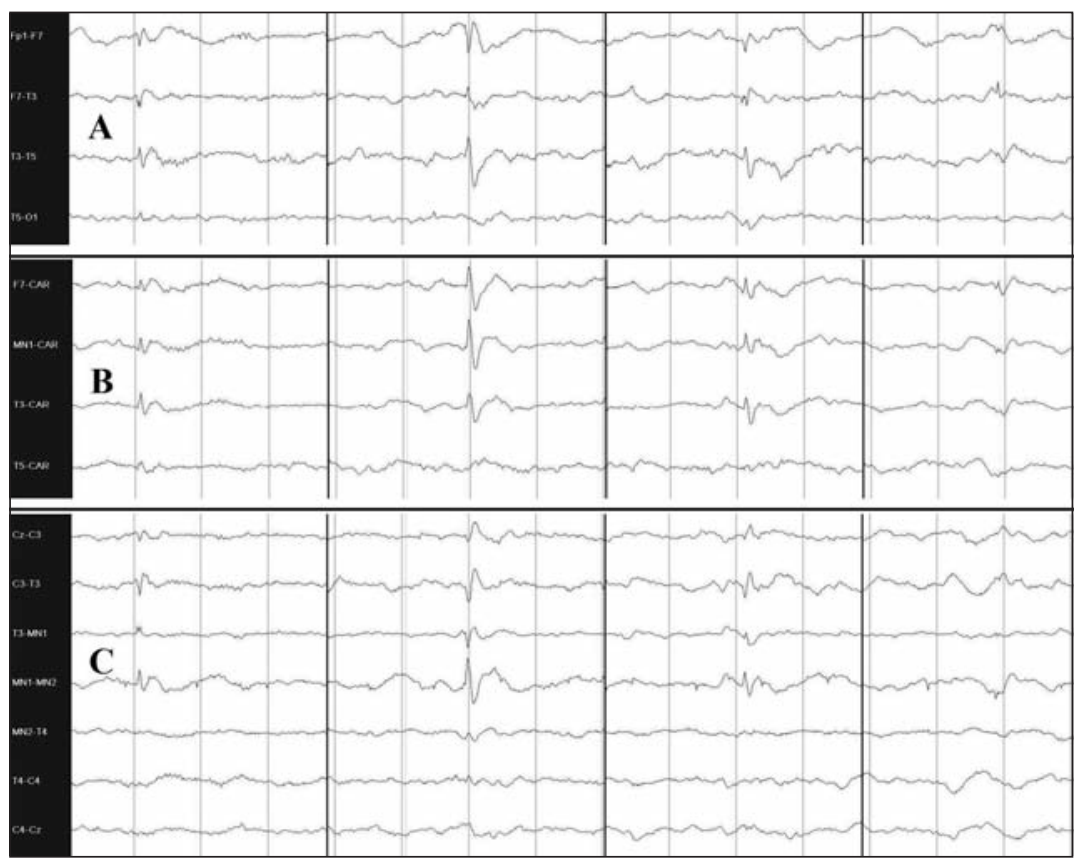

Figure 1: Anterior temporal spike with phase reversal at $F 7$ and shown in three montages, (A) antero-posterior, (B) common average reference $(C A R)$, and $(C)$ coronal with mandibular notch $(M N)$ electrodes. Notice that the spike is better illustrated when $M N$ electrode was used. (A = low frequency filter [LFF] $0.3 \mathrm{sec}$, high frequency filter [HFF] $70 \mathrm{~Hz}$, Sensitivity $7.5 \mu \mathrm{V} / \mathrm{mm} / \mathrm{B \& C}=\mathrm{LFF} 0.3 \mathrm{sec}, \mathrm{HFF} 70 \mathrm{~Hz}$, Sensitivity $10 \mu \mathrm{V} / \mathrm{mm}$ ). 


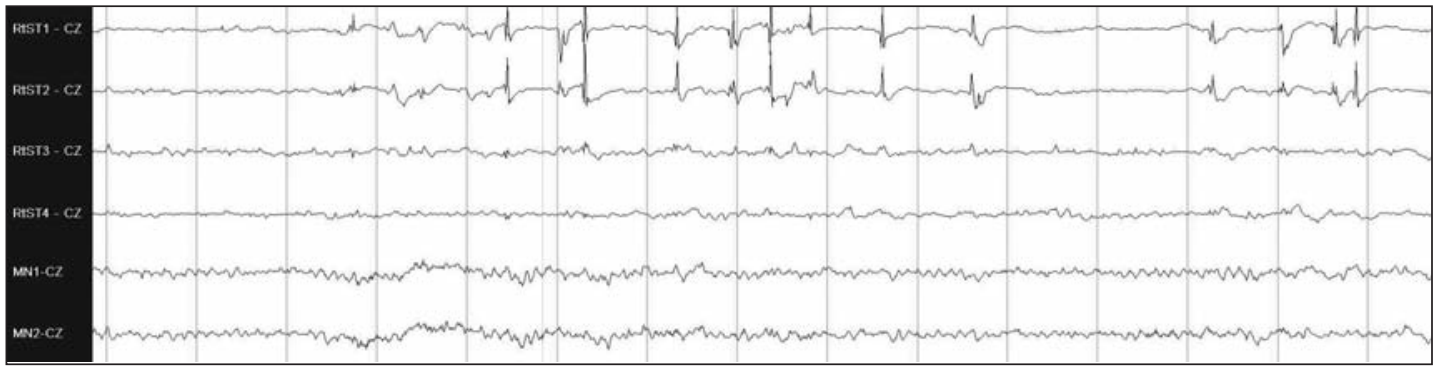

Figure 2: Right anterior temporal spikes on subdural electrodes (top channels) using referential montage. Simultaneous scalp recording (bottom 2 channels) did not reveal the interictal spikes. (low frequency filter [LFF] 0.1 sec, high frequency filter [HFF] $70 \mathrm{~Hz}$, Sensitivity $100 \mu \mathrm{V} / \mathrm{mm}$ for subdural channels / LFF $0.3 \mathrm{sec}$, HFF $70 \mathrm{~Hz}$, Sensitivity 20 $\mu \mathrm{V} / \mathrm{mm}$ for scalp channels).

occipitotemporal junctions) temporal lobe structures ${ }^{17}$. It is important to note that scalp electrodes detect activity generated from a large cortical surface whereas subdural or depth electrodes will detect potential changes which occur over only a few millimeters of cortex. Interictal spikes not otherwise seen on scalp EEG can be easily identified with invasive electrodes (Figure 2). Invasive electrodes are more sensitive in detecting spikes and seizures from a localized area but may miss epileptiform activity which occurs in adjacent regions only a short distance away.

Indications for invasive recording include lack of seizure lateralization and/or localization, seizure onset discrepant with other data (clinical, EEG, and/or imaging), and relation of seizure localization to eloquent cortex. Advantages of invasive monitoring in TLE include improved spatial resolution and increased sensitivity (early signal), no attenuation from scalp and skull, reduced ictal muscle and movement artifact, and utility for direct electrical stimulation of cortex ${ }^{15}$. Disadvantages include limited cortical sampling or risk for sampling error (tunnel vision), and risk of significant complications $(2-3 \%)^{15}$. These complications include aseptic meningitis (subdural more than depth electrodes), cerebrospinal fluid leak, cerebral edema, brain contusion, scalp infection, and bleeding (depth more than subdural electrodes $)^{15}$.

Foramen ovale electrodes are occasionally used to record from mesial temporal structures without requiring penetration of the skull ${ }^{18}$. A multi-contact flexible electrode is placed in the ambient cistern with the aid of a needle inserted through the foramen ovale. These electrodes are not as close to hippocampal structures as intracerebral electrodes and do not allow as large a recording field as grids and strips but detect mesial temporal EEG discharges better than sphenoidal and scalp electrodes ${ }^{18}$.

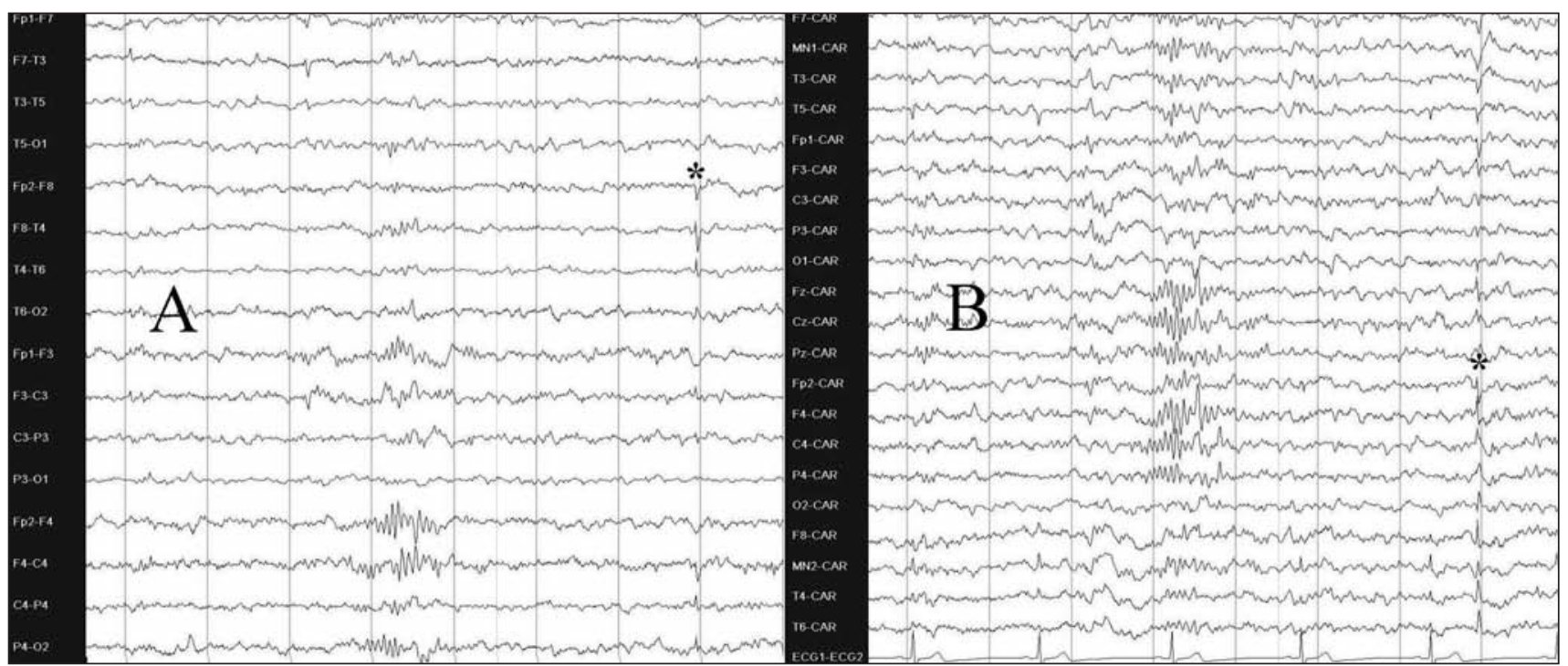

Figure 3: Benign epileptiform transients of sleep (BETS) maximally expressed on the right temporal region on antero-posterior (AP) bipolar montage (A). Their low voltage and wide field is best seen on common average reference $(C A R)(B)$. Notice the central spindles of sleep seen in both figures. ( $A \& B=L F F 0.3 \mathrm{sec}, \mathrm{HFF} 70 \mathrm{~Hz}$, Sensitivity $3 \mu \mathrm{V} / \mathrm{mm})$. 


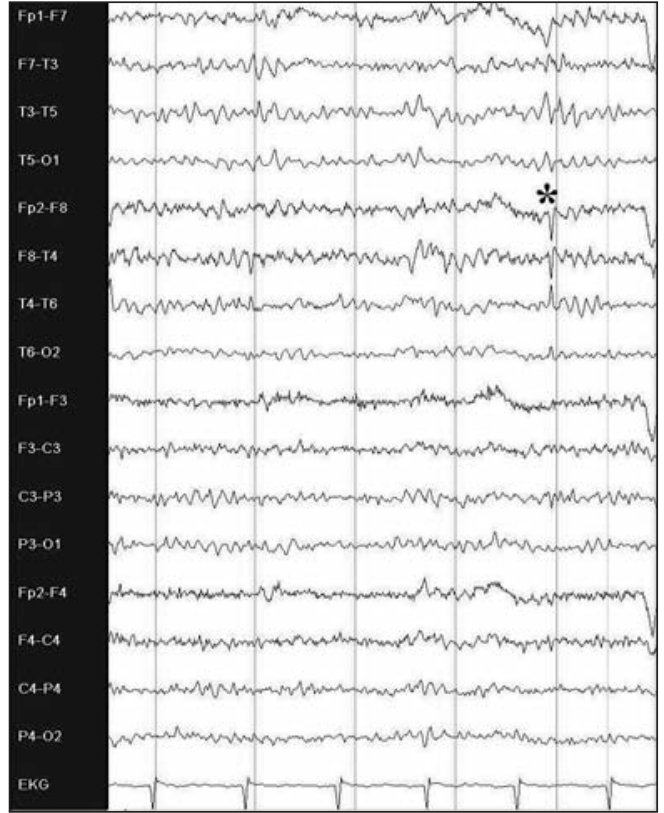

Figure 4: Wicket spikes involving T4 on AP bipolar montage. Notice that there is no after coming slow wave or disruption in the background rhythm. (LFF $0.3 \mathrm{sec}, \mathrm{HFF} 70 \mathrm{~Hz}$, Sensitivity $5 \mu \mathrm{V} / \mathrm{mm}$ ).

When extracranial recordings are equivocal, foramen ovale electrodes offer a less invasive alternative to a more complete intracranial evaluation or can be used in association with grids and strips.

\section{Artefacts}

The differentiation of artefacts from EEG abnormalities can be problematic on video-EEG recordings. Unusual artefacts not seen in standard laboratory EEG recording include mechanical artefacts from altered electrode/scalp contact, intermittent lead wire disconnection, body movement, or movement of the electrode cable $\mathrm{e}^{3,16}$. Artefacts produced by rubbing or scratching of the scalp and other rhythmic movements of head or extremity can result in patterns that must be differentiated from ictal discharges. Some artefacts are characteristic of the oroalimentary automatisms of TLE (e.g. rhythmic chewing and swallowing). Rhythmic slow waves can also be due to chipped electrode coating, instability of the electrode scalp interface, and electrode wire movement. A conservative interpretation of unusual or equivocal EEG events is mandatory, particularly when the patient's activity cannot be verified by video review. Interictal sharp waves noted only during active wakefulness should be interpreted with caution. Ictal rhythms should have a recognizable field with typical ictal progression (evolution of rate and voltage), spread to other regions, or postictal slowing ${ }^{3,16}$. Intracranial recordings tend to have fewer physiologic artefacts, such as chewing and eye blinks.

\section{NORMAL EEG VARIANTS}

The differentiation of some normal or benign EEG transients from EEG abnormalities poses a problem in EEG interpretation and its clinical correlation ${ }^{19}$. Normal EEG variants refer to waveforms that mimic epileptiform abnormalities. In TLE, three important normal variants needs to be recognized to avoid confusion; small sharp spikes of sleep, wicket spikes, and psychomotor variant.

Small sharp spikes of sleep, also known as benign epileptiform transients of sleep, are sharp, small waves occurring unilaterally or bilaterally (often asynchronously), especially in the temporal and frontal regions (Figure 3). Benign epileptiform transients of sleep have large, generally sloping fields in contrast to the restricted field of temporal lobe spikes. They are most often noted in adults during light sleep and have no association with TLE.

Wicket spikes have no pathological significance. They occur almost exclusively in adults and are thought to be due to harmonics of two or more waveforms that combine to form pseudospikes. They have a typical arciform, sharply contoured appearance (Figure 4). When temporal wicket spikes have large amplitude, they may appear similar to pathological spikes.

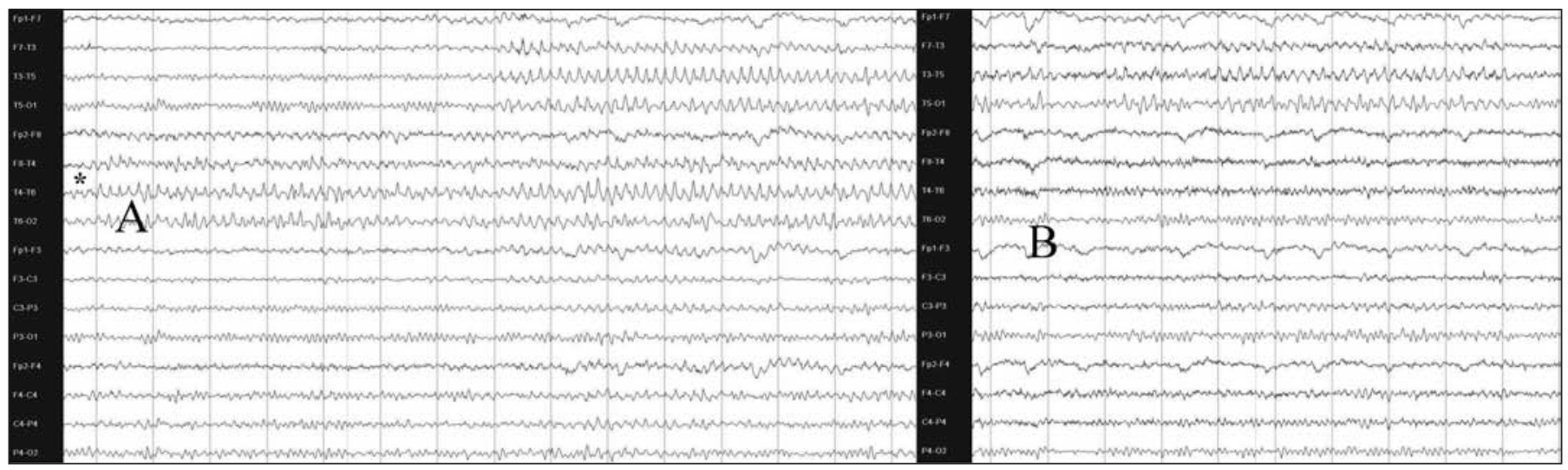

Figure 5: Rhythmic mid-temporal discharge shown on AP bipolar montage. Notice the asymmetry with onset on the right mid temporal region (A), and offset on the other side $(B)$. ( $A \& B=L F F 0.3 \mathrm{sec}, \mathrm{HFF} 70 \mathrm{~Hz}$, Sensitivity $7.5 \mu \mathrm{V} / \mathrm{mm})$. 
However, they can be easily distinguished by their morphology, lack of after coming slow wave, and superimposition on normal background rhythms.

Psychomotor variant (rhythmic mid-temporal discharge) can be confused with an ictal rhythm. This benign rhythm occurs as asymmetrical runs of theta activity primarily in the temporal regions, lasting for a few seconds or as long as 45 seconds (Figure 5). This waveform is a harmonic of two or more rhythms. The waves often have a bifid appearance, start suddenly on one side and last for several seconds before terminating abruptly. The rhythm does not evolve in frequency or amplitude and has no associated clinical symptoms, all of which help in differentiating it from an ictal rhythm ${ }^{19}$.

\section{InTERICTAL EEG AbNormaLITIES}

\section{Background abnormalities}

Focal and regional background slowing (theta and delta) is a nonspecific finding that can be seen in a variety of central disorders, including epilepsy. Persistent focal or regional slowing usually suggests an underlying "structural" abnormality while intermittent slowing is a "functional" abnormality that may indicate an underlying epileptic zone. Lateralized arrhythmic (irregular) delta activity may be found in up to $66 \%$ of patients with TLE and is highly concordant with temporal spiking ${ }^{20}$. These authors concluded that their lateralizing value is similar to that of temporal spiking. Temporal intermittent rhythmic delta activity (TIRDA) is a more specific and accurate interictal indicator of temporal lobe seizures ${ }^{21}$. Trains of TIRDA were found in up to $90 \%$ of patients with MRI demonstrated hippocampal atrophy and TLE ${ }^{22}$. Others confirmed a significant association with mesial rather than lateral $\mathrm{TLE}^{23}$. The delta lateralized to the side of atrophy with accuracy equal to spikes and was never discordant from spikes ${ }^{24}$. These authors concluded that focal TIRDA is a reliable indicator of the side of the epileptogenic focus and is considered an EEG marker of an epileptogenesis that involves the mesial temporal structures. Therefore, focal or regional polymorphic delta in patients with established TLE should not be ignored when assessing the side of ictal onset.

\section{Epileptiform discharges}

The classic interictal EEG abnormality of temporal lobe seizures is a spike (or sharp wave) that has an electronegative peak over the anterior temporal region (F7/F8) as shown in Figure $1^{25}$. Anterior temporal spikes are seen in most $(94 \%)$ patients with medial $\mathrm{TLE}^{26}$. Computer field mapping show that the peak electronegative field of these spikes is anterior and inferior to the standard 10-20 electrode positions (F7/F8); this field accounts for the improved sensitivity of the additional nonstandard electrodes described in the EEG recording section ${ }^{27}$. Most patients with sphenoidal maximum spikes have hippocampal originating seizures ${ }^{28}$. Sphenoidal spikes are also seen in temporal neocortical and orbital frontal onset seizures ${ }^{28}$. Thus, while sphenoidal maximum spikes are sensitive to mesial TLE, they are not highly specific. Mid temporal (T3/T4) and posterior temporal (T5/T6) spikes are more likely to originate from the temporal neocortex.

Sleep increases the spike frequency in TLE where spike foci unapparent during wakefulness may be found ${ }^{29}$. In young patients, a natural sleep recording is always superior to a drug induced sleep as spike activation may occur mainly in the lighter stages of sleep ${ }^{30}$. Sleep deprivation is therefore recommended to achieve this goal, however, this procedure should not be requested routinely because of the possible risk of seizure provocation.

It seems intuitively obvious that patients with a unifocal or very strongly unilaterally predominant interictal temporal spikes have a strong likelihood of ictal onset from the same temporal region $^{31}$. Several studies have demonstrated a significant correlation between the side of ictal onset and the side with a preponderance of interictal spikes ${ }^{32,33}$. This concordance is best if unilateral hippocampal atrophy is associated ${ }^{33}$. Although many publications support the value of the site of maximum interictal spike frequency for predicting the site of ictal onset, a predominant lateralized surface interictal finding may still incorrectly predict the temporal lobe of ictal origin. Ictal recording is therefore recommended before surgical management is considered ${ }^{34}$. Spikes associated with TLE are not always confined to one temporal lobe. Up to $30 \%$ of patients

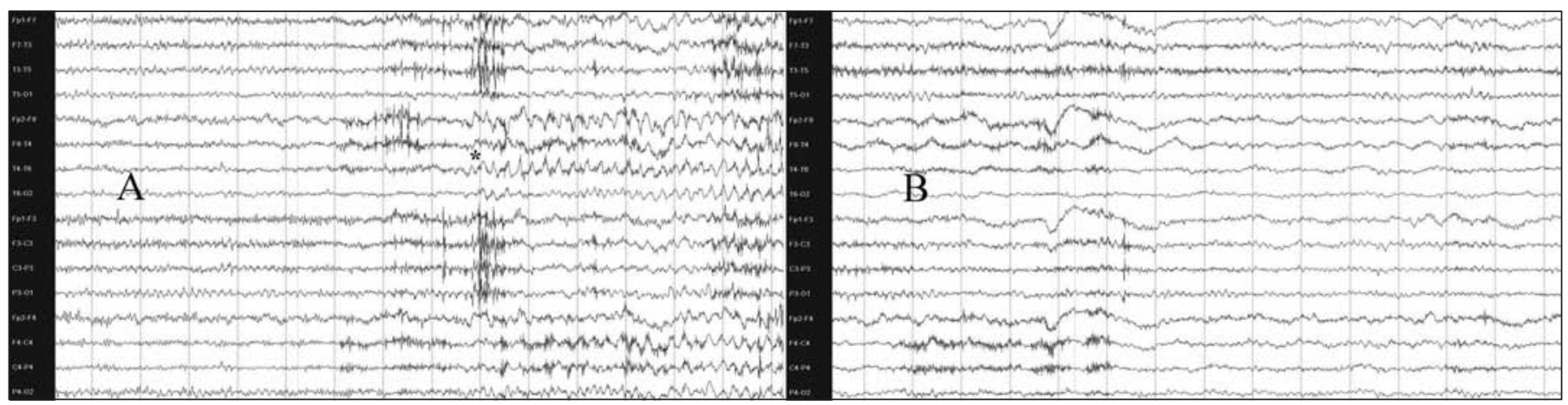

Figure 6: Ictal rhythm in the theta range with right anterior temporal onset (A) on AP bipolar montage. In the postictal phase (B) lateralized polymorphic delta rhythm is shown 45 seconds from seizure offset. (A = LFF $0.3 \mathrm{sec}, \mathrm{HFF} 70 \mathrm{~Hz}$, Sensitivity $5 \mu \mathrm{V} / \mathrm{mm} / \mathrm{B}=\mathrm{LFF} 0.3 \mathrm{sec}, \mathrm{HFF} 70 \mathrm{~Hz}$, Sensitivity $7.5 \mu \mathrm{V} / \mathrm{mm}$ ). 


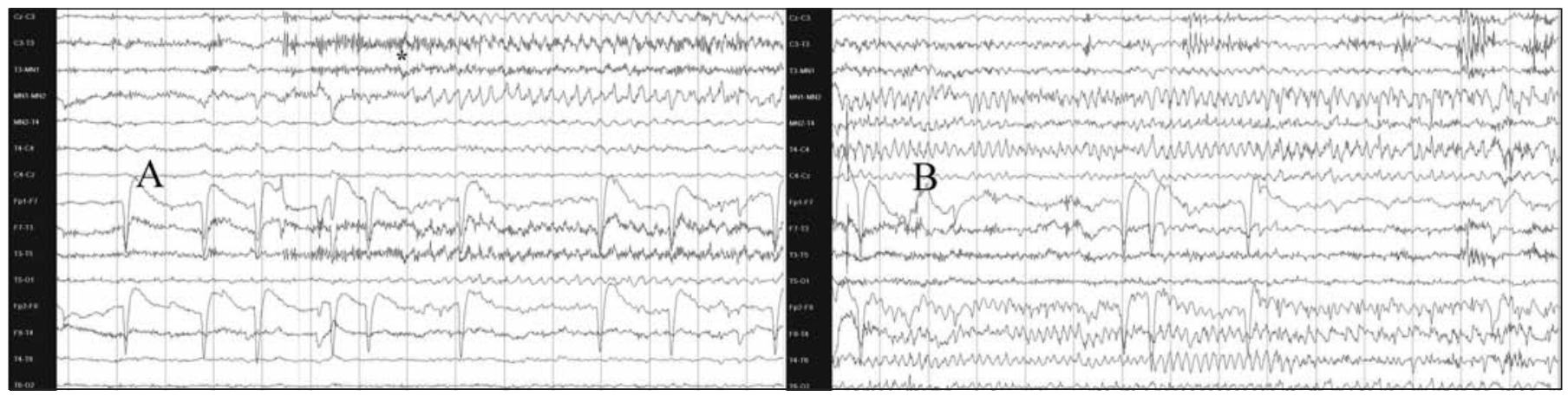

Figure 7: Ictal scalp rhythm in the theta range is shown with left mandibular notch onset (A) on coronal montage. After 45 seconds, the seizure switches to the right side $(B)$. The patient became seizure free after left temporal resection with normal neuropathology. $(A \& B=L F F \quad 0.3$ sec, $H F F 35 H z$, Sensitivity $10 \mu \mathrm{V} / \mathrm{mm}$ ).

with TLE demonstrate independent bitemporal discharges, particularly when prolonged telemetry recording is used ${ }^{3}$. These patients could still have unilateral pathology and subsequent successful surgical outcome, particularly when the neuroimaging abnormality and ictal events were concordant. Bisynchronous spike waves are occasionally encountered in patients with focal temporal EEG abnormalities ${ }^{35,36}$. The mechanism of production of these diffuse waves is unclear; however, their presence should not deter the search for a surgically resectable focus ${ }^{36}$.

\section{ICTAL EEG AbNORMALities}

\section{Scalp recorded rhythms}

Ictal EEG recording is a major component of presurgical evaluation ${ }^{37}$. Ictal rhythms include background attenuation, startstop-start phenomenon, irregular 2-5 Hz lateralized activity, 5-10 $\mathrm{Hz}$ sinusoidal waves or repetitive epileptiform potentials (Figures 6,7). As well, no or minimal EEG change may be noted (see later section on invasive EEG recording). The multiple scalp EEG expressions of temporal lobe originating seizures are likely a consequence of several integrated factors including the location of ictal onset (mesial vs. lateral), the underlying pathology (hippocampal sclerosis or atrophy vs. neoplasm), routes and speed of seizure propagation ${ }^{38}$. Factors that can alter seizure propagation and therefore the EEG expression include the state of the patient (wake vs. sleep), antiepileptic drug use, and whether the patient had unifocal vs. multifocal epilepsy.

Focal or regional background attenuation as the initial EEG change of scalp-recorded temporal lobe seizure is seen in up to $25 \%$ of seizures ${ }^{39}$. In a smaller percentage $(13 \%)$, the seizure may appear to start, stop, and restart "start-stop-start phenomenon" 40 . The initial "start" typically has a more restricted field than the restart. If the initial start is overlooked, the restart may be misinterpreted as the actual seizure onset and therefore considered non-localizing, while in fact the initial start is typically focal and localizing.

Other initial ictal EEG features of TLE include sinusoidal waves and repetitive epileptiform potentials (47\% of cases). As seizures evolve, there is a change in frequency (usually an increase) with spread to other EEG channels as a result of seizure propagation. Only in the final stages of the seizures is there a decrease in wave frequency ${ }^{39}$. A lateralized ictal change of irregular polymorphic $2-5 \mathrm{~Hz}$ temporal rhythm is most often associated with TLE originating from the neocortex ${ }^{41}$. A lateralized ictal change of rhythmic $5-10 \mathrm{~Hz}$ sharp activity, maximally at F7/F8 or sphenoidal electrode position is most commonly seen in hippocampal onset TLE (Figures 6,7). This rhythm usually occurs within 30 seconds of seizure onset and has high specificity for hippocampal onset ${ }^{41-43}$. However, this rhythm is not directly caused by seizures in the hippocampus (see later section on invasive EEG recording).

Another abnormality of possible significance is the increased heart rate just before the onset of ictal EEG discharges ${ }^{44}$. This phenomenon is associated with EEG pattern of TLE originating from the mesial structures, suggesting activation of the neuronal circuits involved in sympathetic regulation.

There are no prospective studies to answer the question of how many ictal recordings should be obtained in a given patient for definite lateralization and localization of TLE. Patients with unilateral interictal spikes were much more likely to have all seizures "concordant" than patients with bilateral interictal spikes $^{45}$. Among the group of patients with "conflicting" seizures, all patients demonstrated the conflicting seizures by the fourth recorded seizure. These results were similar to those of Blum who determined, using a statistical modeling technique that five recorded seizures were required to have a $95 \%$ chance of avoiding a conflicting seizure and only four seizures were needed if all interictal spikes were unilateral ${ }^{46}$.

Potential difficulties with scalp recordings include an inability to interpret the EEG because of excessive muscle and movement artifacts, a paucity of unequivocal changes, interobserver disagreement, and false lateralization. The overall accuracy of scalp lateralization ranges between 60 to $83 \%{ }^{47}$. Other causes of such errors include temporal 'plus' epilepsy and extratemporal originating seizure. Temporal 'plus' epilepsy is characterized by temporal lobe seizures that also involve the neighboring structures such as the orbitofrontal cortex, insula, frontal and parietal opercula, and temporo-parieto-occipital junctions. These are currently most accurately identified by invasive EEG monitoring but scalp EEG can be useful in differentiating TLE from temporal 'plus' epilepsy ${ }^{47}$. In temporal 'plus' epilepsy, the interictal EEG more frequently exhibited 

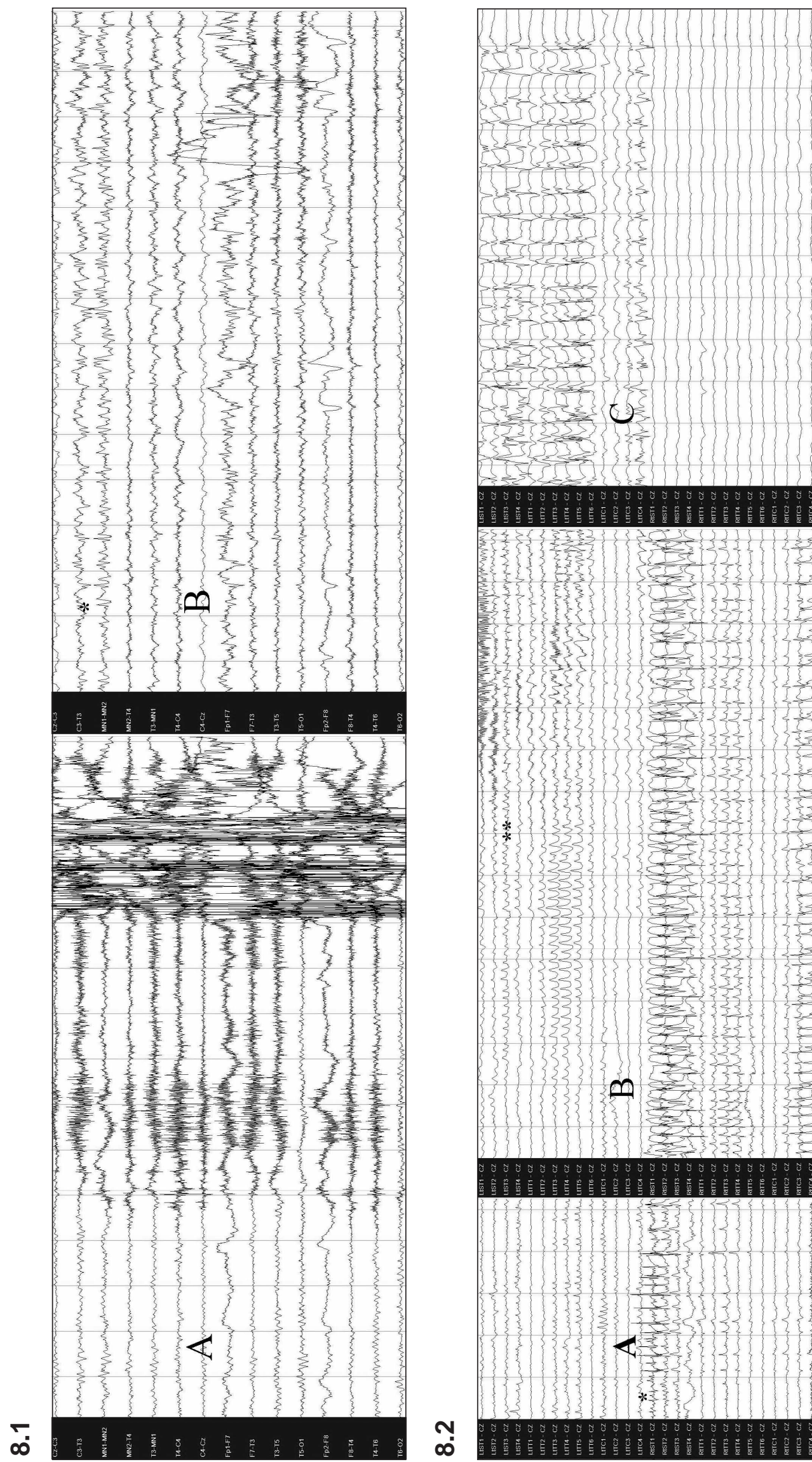

ปี

ฐัฐ

혼

ปั

$\cong \widetilde{\Sigma}$

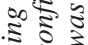

芒

胥

3

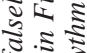

욜ำ

:

5.

$\approx \approx$

$\therefore$ 官:

这?

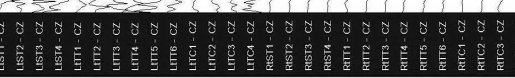

$\therefore \sqrt{3} \overline{8}$

ฐิ

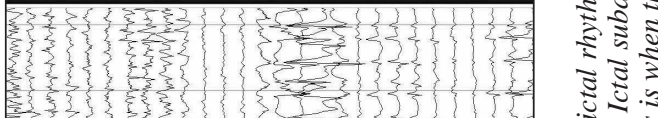
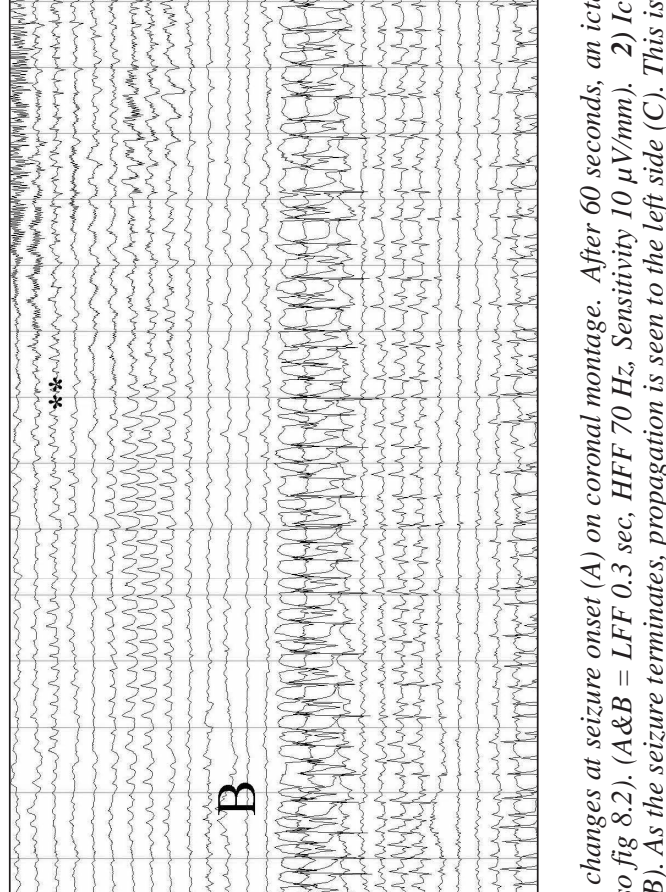

छे仓

造:

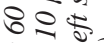

ब.

2.

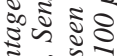

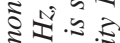

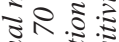

还

8.

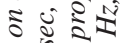

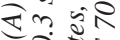

可这

ะ 11 造

เป⿱乛龰)

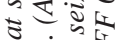

บิษ

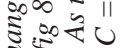

I 0.0

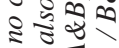

इ

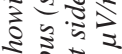

के 홍ำ

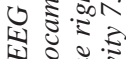

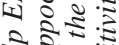

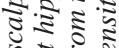

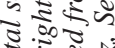



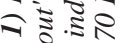

$\ddot{\infty} \cong \bar{c}$

空章这

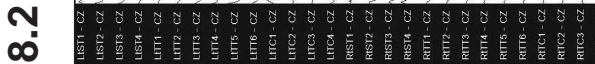

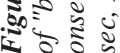


bilateral or precentral abnormalities, while the ictal EEG more frequently suggested early involvement of the anterior frontal, temporo-parietal and central regions.

Occasionally, a seizure starts on one side but terminates ipsilaterally as the seizure propagates to the other temporal lobe (Figure 7). False lateralization of ictal scalp EEG can also be rarely secondary to the so called "burnt out hippocampus"48. Severe advanced hippocampal atrophy and sclerosis results in failure of ipsilateral neocortical propagation and recognition on scalp EEG ipsilaterally ${ }^{49}$. Several seconds after seizure onset, the ictal discharge appears on the contralateral side resulting in false lateralization (Figure 8). Therefore, the ictal rhythm cannot be used in isolation for definite ictal localization.

\section{Intracranial recorded rhythms}

Scalp EEG incompletely records many aspects of intracranial activity $^{50}$. The sensitivity, specificity, and interobserver reliability of scalp ictal EEG for localization of the epileptogenic zone have been disputed. If presurgical surface EEG recording, with additional non-standard EEG electrodes (e.g. sphenoidal or mandibular notch electrodes) cannot provide an adequate answer, invasive intracranial techniques (primarily subdural or intracerebral electrode recordings) can be used to improve localization of the epileptogenic zone ${ }^{51}$. Intracranial EEG directly records paroxysmal activity with high sensitivity, relatively artefact free recording, and excellent temporal resolution in the order of milliseconds ${ }^{52,53}$. In the pre-MRI era the need for such intracranial recording in the determination of an epileptogenic focus was emphasized at some centers where invasive procedures were required in most patients prior to epilepsy surgery. It is now recognized that in most cases of medial temporal lobe epilepsy, standard EEG combined with sphenoidal electrodes and evidence of mesial sclerosis on MRI, will suffice to define the surgical focus.

In addition to the ictal rhythms described in the scalp EEG section, a low voltage fast $(>20 \mathrm{~Hz})$ rhythm can be seen at the onset of intracranial recorded $\mathrm{TLE}^{53}$. It has been demonstrated using a combination of surface and depth electrodes in patients with TLE that a significant proportion of spatially restricted seizures seen with depth recordings produce no visible changes in surface EEG recordings (Figure 8 ) $^{54}$. Only $19 \%$ of auras and $10 \%$ of subclinical seizures are accompanied by scalp EEG changes and occasionally, seizures produce bilateral scalp abnormalities which are not indicative of the side from which depth recorded seizure activity was initiated ${ }^{54}$. A comprehensive systematic study of scalp-intracranial ictal EEG findings in TLE was reported by Pacia and Ebersole ${ }^{55}$. Simultaneous scalp electrodes, mesial temporal depth probes, subdural strip electrodes, and subdural grids were used. Patients with seizures beginning at the hippocampal contacts of the depth electrode had ictal rhythms recorded from the mesial subdural strip electrodes. Simultaneous scalp recordings showed either no change or a diffuse disruption of background. Only when the seizure spread to the basal and inferolateral subdural contacts did a $6-7 \mathrm{~Hz}$ rhythm appear in the inferior and standard scalp electrodes. The authors concluded that a scalp recorded 5-9 Hz rhythm is highly associated with hippocampal onset seizures but is not a direct manifestation of hippocampal ictal activity. This characteristic rhythm appears to reflect recruitment of adjacent temporal cortex and cortico-hippocampal interactions as seizures initiated in the neocortex did not demonstrate this scalp pattern until the hippocampus became involved. Seizures confined to the mesialbasal cortex produce a different scalp rhythm that is manifest on the scalp as a $5 \mathrm{~Hz}$ rhythm maximally expressed, perhaps unexpectedly, at the vertex. This rhythm is of opposite polarity from that seen with the intracranial basal electrodes and although seemingly unlateralized, is in fact quite localizing because of the particular cortical orientation required for its generation ${ }^{55}$. The intracranial correlate of this seizure type was a focal or regional fast rhythm (beta frequency or higher). Such high frequency changes were never seen with scalp recordings in this study. The corresponding scalp recordings consisted of a high voltage, irregular, delta frequency pattern. A further study showed that the location of seizure onset in the temporal lobe was related to the degree of hippocampal pathology ${ }^{56}$. Marked hippocampal atrophy and high grade hippocampal sclerosis were both associated with initial ictal discharge restricted to the hippocampus on depth recording ${ }^{56}$.

Other authors studied the ictal onset of TLE secondary to other pathologies, such as temporal dysembryoplastic neuroepithelial tumor, using invasive subdural EEG recording 57. These patients frequently had more than one ictal onset zone, which were detected more frequently in the tissue adjacent to the tumor rather than within the tumor or in the mesial temporal structures. Such accurate localization of the ictal onset is critical in dysembryoplastic neuroepithelial tumor patients as the surgical outcome was better when all ictal onset zones were completely resected ${ }^{57}$.

\section{Postictal EEG Abnormalities}

Excess muscle and movement artefacts may obscure the EEG features of scalp recordings resulting in difficulties in identifying the seizure onset. Several postictal features are described in TLE including polymorphic delta activity, regional attenuation, or activation of focal spikes. Of these, regional delta was most frequently observed by Kaibara et al in $57 \%$ of patients with scalp EEG recorded seizures ${ }^{58}$. Other authors identified lateralized delta in up to $69 \%$ of postictal EEGs ${ }^{26}$. Postictal change, whatever its nature appeared principally or exclusively ipsilateral to the side of seizure onset in all cases (Figure 6B). In the Williamson et al study of patients rendered seizure-free after a temporal lobectomy, $67 \%$ of patients had lateralized postictal slowing that was concordant to the side of seizure origin in $100 \%$ of instances ${ }^{26}$. Similarly, Walczak et al found that when postictal changes were present and could be lateralized (50\% of patients), these findings were concordant with the side of seizure onset in 96-100\% of patients who were at least two years seizure-free after surgery ${ }^{43}$. The postictal changes may be situated principally over the region most intensely involved in the seizure, which may not always coincide with the region of seizure onset. We evaluated the relationship of postictal delta to the side of seizure onset in patients with TLE by reviewing postictal EEGs with the EEGers blind to clinical and EEG data ${ }^{59}$. Lateralized postictal delta was present in $64 \%$ of all ictal EEGs and in at least one record from 22 patients $(76 \%)$. The two EEGers agreed on the postictal EEG findings in 73 of the 80 seizures (kappa=0.88). Lateralized postictal delta, when present, was concordant with the side of surgery in $96 \%$ of the EEGs. The results confirmed 
that lateralized postictal delta strongly predicts the side of seizure onset in TLE. In another analysis, the postictal EEG features of simple partial (SP) seizures were compared to those of complex partial (CP) and secondary generalized (SG) seizures in patients with documented TLE ${ }^{60}$. Postictal delta was identified in $55 \%, 85 \%$, and $100 \%$ of postictal EEG segments of SP, CP, and SG seizures respectively. Unilateral postictal delta was identified in $36 \%, 76 \%$, and $44 \%$ of postictal EEG segments of $\mathrm{SP}, \mathrm{CP}$, and SG seizures respectively. The EEGs of the partial seizures (SP and CP) were seven times more likely to reveal unilateral PDA when compared to the EEGs of SG seizures, however, unilateral postictal delta was more likely seen in the $\mathrm{CP}$ group. Unilateral postictal delta was ipsilateral to the side of temporal lobectomy in $94 \%$ of EEGs. These findings confirm the potentially important role of postictal EEG in seizure lateralization, particularly if the ictal rhythm was missed or obscured. If polymorphic delta activity emerges in the EEG, it should raise the suspicion of a preceding ictal event.

\section{Summary And Conclusions}

Scalp EEG is needed for accurate lateralization and localization of the seizure onset in TLE. Important EEG background abnormalities include TIRDA, which is a reliable indicator of epileptogenesis that involves the mesial temporal structures. Standard EEG can detect up to $58 \%$ of all interictal spikes, therefore, additional "non-standard" electrodes can be used to further evaluate the EEG abnormalities. Ictal rhythms in TLE includes background attenuation, start-stop-start phenomenon, irregular 2-5 Hz lateralized activity, and $5-10 \mathrm{~Hz}$ sinusoidal waves or repetitive epileptiform potentials. Postictal delta can be lateralized in $60 \%$ of patients with TLE and is concordant with the side of seizure onset in most patients. Invasive EEG is needed in patients with atypical clinical semiology or normal imaging studies. Invasive electrodes can be implanted on the surface of the brain or stereotactically to allow sampling from multiple medial and lateral temporal lobe structures. Electroencephalography abnormalities should be correlated with the clinical and imaging data as accurate identification of the seizure origin is more likely to be achieved if focal EEG onset and MRI findings were concordant with the clinical semiology.

\section{ACKNOWLEDGMENTS}

The authors thank Ms. Meredith Sadler for her preparation of illustrations.

\section{REFERENCES}

1. Sundaram M, Sadler RM, Young GB, Pillay N. EEG in epilepsy: current perspectives. Can J Neurol Sci. 1999;26:255-62.

2. Jan MM. Assessment of the utility of pediatric electroencephalography. Seizure. 2002;11(2):99-103.

3. Sadler M, Desbiens R. Scalp EEG in temporal lobe epilepsy surgery. Can J Neurol Sci. 2000;27:1:22-8.

4. Jan MM, Girvin J. Seizure semiology: value in identifying seizure origin. Can J Neurol Sci. 2008;35:22-30.

5. Jasper H. The ten-twenty electrode system of the International Federation. Electroencephalogr Clin Neurophysiol. 1958;10: 371-5.

6. Sadler RM, Goodwin J. Multiple electrodes for detecting spikes in partial complex seizures. Can J Neurol Sci. 1989;16:326-9.
7. Morris HH, Luders HL, Lesser RP, Dinner DS, Wyllie E. Value of closely spaced electrodes in the localization of epileptiform foci: a study of 26 patients with complex partial seizures. Electroencephalogr Clin Neurophysiol. 1987;63:107-11.

8. Nuwer MR. Recording electrode site nomenclature. J Clin Neurophysiol. 1987;4:121-33.

9. Silverman D. The anterior temporal electrode and the ten-twenty system. Electroencephalogr Clin Neurophysiol. 1960;12:735-7.

10. Kanner AM, Ramirez L, Jones JC. The utility of placing sphenoidal electrodes under the foramen ovale with fluoroscopic guidance. J Clin Neurophysiol. 1995;12:72-81 .

11. Sperling MR, Engel J. EEG from the temporal lobes: a comparison of ear, anterior temporal, and nasopharyngeal electrodes. Ann Neurol. 1985;17:510-3.

12. Sperling MR, Mendius JR, Engel J. Mesial temporal spikes: a simultaneous comparison of sphenoidal, nasopharyngeal, and ear electrodes. Epilepsia. 1986;27:81-6.

13. Binnie CD, Marston D, Polkey CE, et al. Distribution of temporal spikes in relation to the sphenoidal electrode. Electroencephalogr Clin Neurophysiol. 1989;73:403-9.

14. Ives JR, Drislane FW, Schacter SC, et al. Comparison of coronal sphenoidal versus anteroposterior temporal montage in the EEG recording of temporal lobe seizures. Electroencephalogr Clin Neurophysiol. 1996;98:417-21.

15. Dubeau F, McLachlan RS. Invasive electrographic recording techniques in temporal lobe epilepsy. Can J Neurol Sci. 2000;27 (1):29-34

16. Noachtar S, Rémi J. The role of EEG in epilepsy: a critical review. Epilepsy Behav. 2009;15(1):22-33.

17. Maillard L, Vigal JP, Gavaret M, et al. Semiologic and electrophysiologic correlations in temporal lobe seizure subtypes. Epilepsia. 2004;45(12):1590-9.

18. Nilsson D, Fohlen M, Jalin C, Dorfmuller G, Bulteau C, Delalande O. Foramen ovale electrodes in the preoperative evaluation of temporal lobe epilepsy in children. Epilepsia. 2009;50(9): 2085-96.

19. Santoshkumar B, Chong JJ, Blume WT, et al. Prevalence of benign epileptiform variants. Clin Neurophysiol. 2009;20:856-61.

20. Koutroumanidis M, Martin-Migel C, Hennessy MJ, et al. Interictal temporal delta activity in temporal lobe epilepsy: correlations with pathology and outcome. Epilepsia. 2004;45(11):11351-67.

21. Geyer JD, Bilir E, Faught RE, Kuzniecky R, Gilliam F. Significance of interictal temporal lobe delta activity for localization of the primary epileptogenic region. Neurology. 1999;52:202-5.

22. Gambardella A, Gotman J, Cendes F, Andermann F. Focal intermittent delta activity in patients with mesiotemporal atrophy: a reliable marker of the epileptogenic focus. Epilepsia. 1995;36:122-9.

23. Di Gennaro G, Quarato PP, Onorati P, et al. Localizing significance of temporal intermittent rhythmic delta activity (TIRDA) in drug-resistant focal epilepsy. Clin Neurophysiol. 2003;114:70-8.

24. Blume WT, Borghesi JL, Lemieux JF. Interictal indices of temporal lobe origin. Ann Neurol. 1993;34:703-9.

25. Klass D. Electroencephalographic manifestations of complex partial seizures. In: Penry JK, Daly DD, editors. Advances in neurology. New York: Raven Press; 1975. p. 113-40.

26. Williamson PD, French JA, Thadani VM. Characteristics of medial temporal lobe epilepsy: II. Interictal and ictal scalp electroencephalography, neuropsychological testing, neuroimaging, surgical results, and pathology. Ann Neurol. 1993;34: 781-7.

27. Sadler RM, Lemieux JF, Blume WT. Potential fields of anterior temporal spikes. Electroencephalogr Clin Neurophysiol. 1984; 58:47-8.

28. Marks DA, Kratz A, Booke J, et al. Comparison and correlation of surface and sphenoidal electrodes with simultaneous intracranial recording: an interictal study. Electroencephalogr Clin Neurophysiol. 1992;82:23-9.

29. Malow BA, Kushwaha R, Lin X, et al. Relationship of interictal epileptiform discharges to sleep depth in partial epilepsy. Electroencephalogr Clin Neurophysiol. 1997;102:20-6.

30. Jan MM, Aquino MF. The use of chloral hydrate in pediatric electroencephalography. Neurosciences. 2001;6(2):99-102. 
31. Quesney LF, Risinger MW, Shewmon DA. Extracranial EEG evaluation. In: Engel J, editor. Surgical treatment of the epilepsies. New York: Raven Press; 1993. p. 173-95.

32. Holmes MD, Dodrill CB, Wilensky AJ, et al. Unilateral focal preponderance of interictal epileptiform discharges as a predictor of seizure origin. Arch Neurol. 1996;53:228-32.

33. Cendes F, Li LM, Watson C, Andermann F, Dubeau F, Arnold DL. Is ictal recording mandatory in temporal lobe epilepsy? Arch Neurol. 2000;57:497-500.

34. Engel J, Wiebe S, French J, et al. Practice parameter: temporal lobe and localized neocortical resections for epilepsy. Neurology. 2003;60:538-47.

35. Gabor A, Ajmone-Marsan C. Co-existence of focal and bilateral diffuse paroxysmal discharges in epileptics: a clinical electrographic study. Epilepsia. 1969;10:453-72.

36. Sadler RM, Blume WT. Significance of bisynchronous spike-waves in patients with temporal lobe spikes. Epilepsia. 1989;30: 143-6.

37. Engel J. Update on surgical treatment of the epilepsies. Neurology. 1993;43:1612-7.

38. Lieb J, Dasheiff R, Engel J. Role of the frontal lobes in the propagation of mesial temporal lobe seizures. Epilepsia. 1991; 32:822-37.

39. Blume WT, Young GB, Lemieux JF. EEG morphology of partial epileptic seizures. Electroencephalogr Clin Neurophysiol. 1984; 57:295-302.

40. Atalla N, Abou-Khalil B, Fakhoury T. The start-stop-start phenomenon in scalp-sphenoidal recordings. Electroencephalogr Clin Neurophysiol. 1996;98:9-13.

41. Ebersole JS, Pacia SV. Localization of temporal lobe foci by ictal EEG patterns. Epilepsia. 1996;37(4):386-99.

42. Vossler DG, Abson Kraemer DL, Knowlton RC, et al. Temporal ictal electroencephalographic frequency correlates with hippocampal atrophy and sclerosis. Ann Neurol. 1998;43: 756-62.

43. Walczak T, Radtke R, Lewis D. Accuracy and interobserver reliability of scalp ictal EEG. Neurology. 1992;42:2279-85.

44. Di Gennaro G, Quarato PP, Sebastiano F, et al. Ictal heart rate increase precedes EEG discharge in drug-resistant mesial temporal lobe seizures. Clin Neurophysiol. 2004;115:1169-77.

45. Sirven JI, Liporace JD, French JA, et al. Seizures in temporal lobe epilepsy. I. Reliability of scalp sphenoidal ictal recording. Neurology. 1997;48:1041-6.

46. Blum D. Prevalence of bilateral partial seizure foci and implications for electroencephalographic telemetry monitoring and epilepsy surgery. Electroencephalogr Clin Neurophysiol. 1994;91: 329-36.
47. Bara C, Barbati G, Minotti L, Hoffman D, Kahane P. Ictal clinical and scalp-EEG findings differentiating temporal lobe epilepsies from temporal 'plus' epilepsies. Brain. 2007;130:1957-67.

48. Mintzer S, Cendes F, Soss J, et al. Hippocampal sclerosis with contralateral temporal scalp ictal onset. epilepsia. 2004;45(7): 792-802.

49. Castro LH, Serpa MH, Valerio RM, et al. Good surgical outcome in discordant ictal EEG-MRI unilateral mesial temporal sclerosis patients. Epilepsia. 2008;49(8):1324-32.

50. Cooper R, Winter AL, Crow HJ, et al. Comparison of subcortical, cortical, and scalp activity using chronic indwelling electrodes in man. Electroencephalogr Clin Neurophysiol. 1965;18:217-28

51. King D, Spencer S. Invasive electroencephalography in mesial temporal lobe epilepsy. J Clin Neurophysiol. 1995;12:32-45.

52. Wendling F, Hernandez A, Bellanger J, Chauvel P, Bartlomei F. Interictal to ictal transition in human temporal lobe epilepsy: insights from a computational model of intracerebral EEG. J Clin Neurophysiol. 2005;22(5):343-56.

53. Jung WY, Pacia SV, Devinsky O. Neocortical temporal lobe epilepsy: intracranial EEG features and surgical outcome. J Clin Neurophysiol. 1999;16(5):419-26.

54. Lieb JP, Walsh GO, Babb TL, et al. A comparison of EEG seizure patterns recorded with surface and depth electrodes in patients with temporal lobe epilepsy. Epilepsia. 1976;17:137-60.

55. Pacia SV, Ebersole JS. Intracranial EEG substrates of scalp ictal patterns from temporal lobe foci. Epilepsia. 1997;38:642-54.

56. Vossler DG, Kraemer DL, Haltiner AM, et al. Intracranial EEG in temporal lobe epilepsy: location of seizure onset relates to degree of hippocampal pathology. Epilepsia. 2004(45(5): 497-503.

57. Soe DW, Hong SB. Epileptogenic foci on subdural recording in intractable epilepsy patients with temporal dysembryoplastic neuroepithelial tumor. J Korean Med Sci. 2003;18:559-65.

58. Kaibara M, Blume WT. The postictal electroencephalogram. Electroencephalogr Clin Neurophysiol. 1988;70:99-104.

59. Jan MM, Sadler M, Rahey SR. Lateralized postictal EEG delta predicts the side of seizure surgery in temporal lobe epilepsy. Epilepsia. 2001;42:402-5

60. Jan MM. The value of postictal electroencephalogram in temporal lobe seizures. Ann Saudi Med. 1999;19:550-3. 